\title{
Markets of information goods facing a strong P2P network
}

Citation for published version (APA):

Yang, S. (2010). Markets of information goods facing a strong P2P network. METEOR, Maastricht University School of Business and Economics. METEOR Research Memorandum No. 037 https://doi.org/10.26481/umamet.2010037

Document status and date:

Published: 01/01/2010

DOI:

10.26481/umamet.2010037

Document Version:

Publisher's PDF, also known as Version of record

\section{Please check the document version of this publication:}

- A submitted manuscript is the version of the article upon submission and before peer-review. There can be important differences between the submitted version and the official published version of record.

People interested in the research are advised to contact the author for the final version of the publication, or visit the DOI to the publisher's website.

- The final author version and the galley proof are versions of the publication after peer review.

- The final published version features the final layout of the paper including the volume, issue and page numbers.

Link to publication

\footnotetext{
General rights rights.

- You may freely distribute the URL identifying the publication in the public portal. please follow below link for the End User Agreement:

www.umlib.nl/taverne-license

Take down policy

If you believe that this document breaches copyright please contact us at:

repository@maastrichtuniversity.nl

providing details and we will investigate your claim.
}

Copyright and moral rights for the publications made accessible in the public portal are retained by the authors and/or other copyright owners and it is a condition of accessing publications that users recognise and abide by the legal requirements associated with these

- Users may download and print one copy of any publication from the public portal for the purpose of private study or research.

- You may not further distribute the material or use it for any profit-making activity or commercial gain

If the publication is distributed under the terms of Article $25 \mathrm{fa}$ of the Dutch Copyright Act, indicated by the "Taverne" license above, 


\section{Maastricht University}

Michael S. Yang

Markets of information goods facing a strong P2P network

$\mathrm{RM} / 10 / 037$

\section{METEOR}

Maastricht University School of Business and Economics

Maastricht Research School of Economics

of Technology and Organization

P.O. Box 616

NL - 6200 MD Maastricht

The Netherlands 


\title{
Markets of information goods facing a strong P2P network
}

\author{
Michael S. Yang \\ July 19, 2010
}

\begin{abstract}
This paper studies a traditional monopolistic market of information goods in the presence of an inherently strong peer-to-peer file-sharing network. Specifically, such a strong network is made possible by a few fanatic users who selflessly contribute to the sharing of files. We find that the most prevalent equilibrium outcome is the one where the firm accommodates the network and competes in price. We establish that coordination failure in the forming of networks is not an issue once we consider a high level of taste heterogeneity and include such fanatic users in the model. We also find possible network-deterring market structures, although these can only happen under limited circumstances. Furthermore, it is not impossible to see the firm and the network co-existing as local monopolies not serving the entire market and therefore not competing. For this market structure to occur, the taste heterogeneity has to be very large. Finally, we find that in all the equilibrium structures, total welfare always decreases in taste heterogeneity and the generic cost factor of downloading.
\end{abstract}

JEL classification: L11; L82; L86.

Keywords: information products; the music industry; piracy; P2P (peer-to-peer) filesharing networks; network externalities; pricing; multi-platform competition.

\section{Introduction}

This paper studies a traditional monopolistic market of information goods in the presence of an inherently strong peer-to-peer file-sharing network. In most of today's P2P file-sharing networks, there are always a few fanatic users who selflessly contribute to the sharing of files by uploading their own files (e.g. music, movies, software, etc.) for other's to download. The model is taken from Herings, Peeters and Yang (2008), but here we look at a different set of parameter values in order to reflect this inherent strength of the $\mathrm{P} 2 \mathrm{P}$ networks.

There are two lines of literature which are related to the online piracy fostered by P2P file-sharing networks. One is the literature on piracy itself, including papers like Novos and Waldman (1984), Liebowitz (1985), Johnson (1985), where the authors study how a firm can react to piracy. The firm generally has three possibilities, do not react at all if piracy poses no real threat, use piracy-deterring pricing, or use piracy-accommodating pricing. The other line of classical literature concerns network externalities, where representative papers include e.g. Katz and Shapiro $(1985,1986)$ and Farrell and Saloner $(1985,1986)$. Conner and 
Rumelt (1991) and Takeyama (1994) combine the two subjects and find that unauthorized reproduction of intellectual property in the presence of consumptive externalities can induce greater firm profits and lead to Pareto improvements.

Recent literature has also studied the phenomenon of P2P in particular. Gayer and Shy (2003) studies how publishers of digitally-stored products can utilize P2P networks to enhance sales of their products sold in the stores. This result follows from the positive consumption externalities in their model. Another way in which firms can benefit from P2P activity is in set-ups with imperfect information about product characteristics. The effects of sampling possibilities due to P2P networks are studied by Peitz and Waelbroek (2006b). The authors show that under sufficient taste heterogeneity and product diversity, the positive effect of downloading on sales due to sampling may compensate the negative effect.

The most relevant existing paper is our previous work Herings et al. (2008), where we developed a two-stage game theoretic model that is also adopted in this paper. The focus there is a monopoly facing a $\mathrm{P} 2 \mathrm{P}$ network with positive but nonlinear externalities. The paper models the interaction of the firm and the P2P file-sharing network as a two-stage game. In the first stage, the firm sets a price for the physical form of the product. Then, in the second stage, after having observed the price set by the firm, consumers simultaneously and independently decide whether to legally buy the physical form of the product or to download the digital form via the $\mathrm{P} 2 \mathrm{P}$ network, or not to acquire the content at all. The consumers are heterogenous with respect to their tastes regarding the physical form and the digital form of the product, and we use the standard Hotelling model to describe their preferences. In their decisions, consumers have to anticipate each others' decisions, since the resulting network size determines the actual costs of downloading. In such circumstances, consumers may fail to coordinate on a Pareto-optimal network size, thereby giving the firm a lot of room to price the network out of the market.

We adopt the same model in this paper, but with one crucial difference on the parameter assumptions. In Herings et al. (2008), there is an assumption on two parameters that leads to the situation where no consumer will join a network if the size of such a network is expected to be 0 . We reverse the relation between these two parameters so that now the consumer who prefers the digital version the most will join a P2P network even if the network is expected to be empty. This is to reflect the realistic situation where there are always some "die-hard" P2P network fanatics who selflessly provide the availability of such networks.

Furthermore, we relax the assumption on the relatively small parameter value of consumers' taste heterogeneity. More specifically, we now allow consumers' taste heterogeneity to be so high that consumers with extreme preferences may not consume the least preferred version of the product even if it is costless. In so doing, we are able to capture more scenarios, including those where the market is partially served by the firm and the network together. We discover that coordination failure in the forming of networks is not an issue once we consider a high level of taste heterogeneity and include such fanatic users in the model. 
In total, we find five possible equilibrium market structures in this paper. Out of the five market structures, the most prevalent is the one where the firm accommodates the network and competes in price. In this case, the firm's profit suffers and the total welfare benefits from the existence of $\mathrm{P} 2 \mathrm{P}$. In the settings of this paper, the firm can still deter the network, but under very limited circumstances when taste heterogeneity is low, the quality of the physical form is high, and the legal enforcement is relatively strict. Think of the newspaper market as an example of this. The firm can also find itself in the position to want to optimally serve the whole market, if the value of its product is significantly higher than that of the pirated version, and the taste heterogeneity is low. Another special market structure is that of a market-sharing case, which happens when taste heterogeneity is just so that the firm chooses to precisely serve the consumers who prefer not to download. Effectively, the firm lets the network form to its maximum size, and then serves the residual demand. Thus, the firm and the network together serve the market fully without competing with each other. Furthermore, there is a possibility where the firm can ignore the network and act as a monopoly, when taste heterogeneity is huge. Yet the firm's profit actually decreases in taste heterogeneity. So does the consumers' surplus. Total welfare, therefore, decreases in taste heterogeneity, and in the cost factor of downloading.

The remainder of the paper is organized as follows. In Section ?? the two-stage model is described in detail. Next, the consumers' choices in the second stage, given the price set by the firm in the first stage, are presented in Section ??. Subsequently, in Section ??, the firm's pricing decision in the first stage, and the resulting market structures, are analyzed. Thereafter, Section ?? gives an overview of the equilibrium outcomes analyzed in Section ?? and provides economic intuitions. Section ?? discusses the comparative statics of the monopoly equilibrium outcome. Finally, Section ?? and 8 conclude and discuss the results.

\section{The model}

The model is taken from Herings et al. (2008), where we consider two forms of the same product. We refer to the legal product sold by the firm (CD or DVD) as the physical form and the P2P version of it as the digital form.

The game has two stages. In stage one, the firm sets a price $p$ for the physical form of the product. Next, in stage two, after having observed the price set by the firm, consumers decide simultaneously and independently whether to purchase the physical form sold by the firm $(S)$, to download the digital form via the P2P network $(N)$, or not to acquire the product at all $(\emptyset)$. There is a continuum of consumers who differ in their relative preference of the physical form over the digital form.

The price $p$ set by the firm and the profile of consumers' choices determine the sales by the firm, $s \in[0,1]$, total quantity of consumers buying the physical form, and the size of the network $n \in[0,1]$, total quantity of consumers who choose to download the digital form from 
the network.

We assume, for simplicity, that the firm has zero costs in production and aims to maximize its profit, given by

$$
\pi=p \cdot s(p)
$$

The utility of a consumer with identity $x \in[0,1]$ is given by

$$
U^{x}(p)= \begin{cases}\beta-\tau x-p & \text { if } S \\ \gamma-\tau(1-x)-C(n(p)) & \text { if } N \\ 0 & \text { if } \emptyset\end{cases}
$$

where $\beta>0$ and $\gamma>0$ represent the basic utility of the physical and the digital form respectively. The identity $x \in[0,1]$ reflects the consumer's relative preference over the two forms. The consumer with identity $x=0$ has a strong preference for the physical form, whereas the consumer with identity $x=1$ has a strong preference for the digital form. For consumers $x \in(0,1)$, the acquisition of one of the forms generates a disutility that depends on the identity $x$ and the parameter $\tau>0$. The parameter $\tau$ captures the amount of heterogeneity in consumers' tastes. Notice that $\beta$ and $\gamma$ capture the objective qualities of the two forms of the products, while $\tau$ is a factor that influences the subjective individual utility a consumer gets from the product. For instance, an illegally downloaded album of MP3 files is of inferior quality to the original CD that it is "ripped" from, but some might actually prefer the MP3 version since it's easier to carry around in the iPod.

Finally, $C(n)$ represents the costs of downloading when the resulting network is of size $n \in[0,1]$. A higher number of users of a network improves the availability of files and hence decreases the standard search costs and downloading time. Therefore, we implement a cost function of downloading that is decreasing and convex in the number of users of the P2P network, ${ }^{1}$

$$
C(n)=\sigma \cdot(1-n)^{2},
$$

where $\sigma>0$ represents the generic cost factor of downloading, incorporating a collection of factors that may affect downloading costs, for instance, the degree of legal enforcement of intellectual property rights. Note that $\sigma$ is identical for every consumer and is independent of the network size.

We impose some assumptions on the consumers' behavior in case of indifference. When a consumer is indifferent between buying from the firm and not acquiring the product or between the firm and the network, she chooses the firm. When she is indifferent between the network and not acquiring the product, she chooses the network. Thus, without loss of

\footnotetext{
${ }^{1}$ The quadratic specification of the cost function for downloading satisfies the qualitative properties just stated, while it preserves analytical tractability of the model.
} 
generality, we assume a linear order of priority of the firm $(S)$ above the network $(N)$, and the network $(N)$ above not acquiring the product $(\emptyset)$.

Next, we impose the following assumptions on the relevant parameters.

Assumption 1. $\beta>\gamma$.

Personal preferences aside, the objective product quality of the original physical form is higher than that of the digital form (which is "ripped" from the original).

\section{Assumption 2. $\gamma>\sigma$.}

There is at least one consumer (namely, the one located at $x=1$ ), who prefers joining $\mathrm{P} 2 \mathrm{P}$ to not consuming at all even if the network is empty. Or to put it differently, the consumer who prefers the digital form the most is always willing to start a network. This implies that there is always a $\mathrm{P} 2 \mathrm{P}$ network, unless the firm charges such a low price that even the consumer located at $x=1$ prefers to buy the physical form, in which case the market is fully served by the firm. In other words, this consumer is always served.

Note that we do not impose any assumptions on the value of $\tau$.

\section{Consumers' choice}

We start the analysis with determining the possible Nash equilibria for each of the subgames. Then, we consider the firm's pricing behavior in the first stage and analyze some interesting subgame-perfect Nash equilibrium outcomes.

Firstly, it is important to clarify three terms that we use in describing the equilibrium solutions of the network size $n \in[0,1]$ : no network, critical-mass network and maximum network. Consider the case where the price set by the firm in stage one is high enough to be consistent with the formation of a P2P network. Due to the positive network externalities and the law of demand, there are typically two equilibrium network sizes. The smaller one is called the critical-mass network and denoted by c.m.. If all the consumers expect that the network size to be like this, such expectations will be self-enforcing. There is one consumer who is indifferent between buying from the firm and downloading via the P2P network and whose choice follows the priority order assumption. All consumers with higher utility from downloading are strictly better off joining the network and all the other consumers are strictly better off at the firm.

Since we have positive downloading externalities, the critical-mass network is destabilized when slightly more consumers, located closest to the indifferent consumer, join it. If all consumers expect that the network is like this, the consumer with the highest value of $x$ buying at the firm will strictly prefer to download, so these expectations are not compatible with a second-stage equilibrium. When this consumer changes to the network, the same process happens to the consumer next to him who now may also strictly prefer to download. 
This process continues until the network size stabilizes at a larger size, called the maximum network, denoted by m.n. that can be supported as a second-stage equilibrium, where the maximal network may be the one with all consumers in it.

The opposite reasoning applies when the critical-mass network is destabilized because slightly less consumers located closest to the indifferent consumer joins the network. Now less and less consumers will want to join the network until the network reaches the size of 0 .

Therefore, there are in total three equilibrium network sizes. The maximum network and the zero network are stable, and the critical-mass network is instable. This gives rise to the concept of coordination failure in network forming. Within our terminology, a network may experience coordination failure only when both the maximum and the critical-mass network exist, given the relevant price. In other words, if for a given price, the critical-mass network does not exist, coordination failure can not occur. In the majority part of the analysis in this paper, the maximum network is the only equilibrium network size. A formal computation of the solutions of the critical-mass and maximum network in the fully- and partially-served markets can be found in the Appendices.

Second-stage equilibrium market structures can differ in two dimensions: the degree to which consumers are served and the platforms that are actively used. Regarding the first dimension, the market can be fully served or partially served as is displayed in Figure 1 for a multi-platformed market. ${ }^{2}$ Regarding the second dimension we can have a multi-platformed
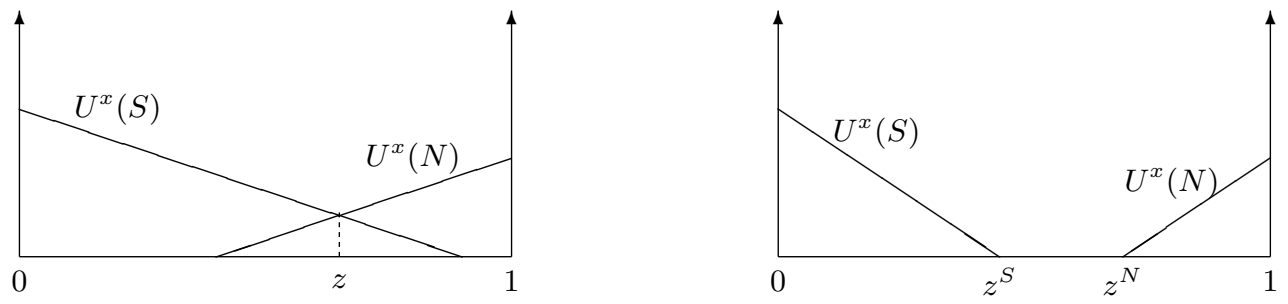

Figure 1: Fully-served market and partially-served market.

market or a single-platformed market with either only the firm or only the P2P network. Note that the market can not be partially served by the store alone due to Assumption 2, i.e. $[S / \emptyset]$ does not exist. For the second stage, we can therefore restrict our attention to the five market structures depicted in Table 1. Observe that, the situation in the graph on the left of Figure 1 is denoted by $[S / N]$; and the one on the right by $[S / \emptyset / N]$.

Which one of these five possible market structures prevails in the second stage depends, apart from the price $p$ set in the first stage, on the parameter values. The set of parameter values determines the group of possible second-stage equilibrium market structures.

Recalling Assumption 2, we have $\gamma>\sigma$, but no restrictions on their relations with $\tau$. Not putting any assumptions on $\tau$ results in three possible scenarios: high heterogeneity scenario

\footnotetext{
${ }^{2}$ Notice that the vertical intercept of the utility level from the network is endogenously determined by the resulting network size. The quantities $z, z^{S}$ and $z^{N}$ are used for the analysis in the appendices.
} 


\begin{tabular}{lccc}
\hline & \multicolumn{2}{c}{ single-platformed } & \\
\cline { 2 - 3 } & firm & network & multi-platformed \\
\hline fully-served & {$[S]$} & {$[N]$} & {$[S / N]$} \\
partially-served & & {$[\emptyset / N]$} & {$[S / \emptyset / N]$} \\
\hline
\end{tabular}

Table 1: All possible market structures in stage two.

$(\tau>\gamma>\sigma)$, medium heterogeneity scenario $(\gamma>\tau>\sigma)$, and low heterogeneity scenario $(\gamma>\sigma>\tau) .{ }^{3}$ These three different scenarios in turn yield very different equilibrium market outcomes in the second stage given the price set by the firm in the first stage. Depending on the level of taste heterogeneity, the market can be fully or partially served, by single or multiple platforms. In the following analysis we will examine the scenarios separately .

Proposition 1. Given the price p set in the first stage by the firm, all second-stage equilibrium market structures in the high heterogeneity scenario $(\tau>\gamma>\sigma)$ are as depicted in Figure 2.

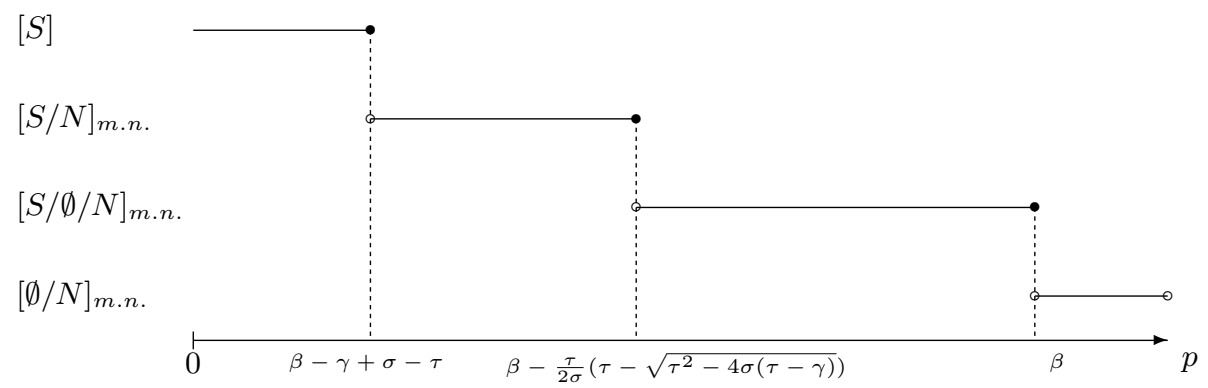

Figure 2: High heterogeneity scenario

The horizontal axis depicts the first-stage price that the firm charges, and the corresponding market structures are shown on the vertical axis. ${ }^{4}$

As the firm raises its price, it gradually loses its market share. When it charges a price lower than $\beta-\gamma+\sigma-\tau$, it fully serves the market alone and deters the formation of the network. Note that if $\beta-\gamma+\sigma-\tau<0$, the market structure $[S]$ will disappear and $[S / N]$ will start at the price of 0 instead. But since this condition does not affect the equilibrium outcome, we shall not go through the trouble to treat it with separate scenarios. At a price between $\beta-\gamma+\sigma-\tau$ and $\beta-\frac{\tau}{2 \sigma}\left(\tau-\sqrt{\tau^{2}-4 \sigma(\tau-\gamma)}\right)$, the firm accommodates the network and together they serve the market fully. At a price between $\beta-\frac{\tau}{2 \sigma}\left(\tau-\sqrt{\tau^{2}-4 \sigma(\tau-\gamma)}\right)$ and $\beta$, it ignores the network and acts as a monopoly to serve its own monopolistic market share. In this case the market is partially served. Again, if $\beta-\frac{\tau}{2 \sigma}\left(\tau-\sqrt{\tau^{2}-4 \sigma(\tau-\gamma)}\right)<0$,

\footnotetext{
${ }^{3}$ We ignore the specific analysis of boundary cases where $\tau=\gamma$ or $\tau=\sigma$, since they produce degenerate results without additional insights.

${ }^{4}$ The proofs of this and the two subsequent propositions can be found in the Appendices.
} 
both $[S]$ and $[S / N]$ will disappear from the graph, and $[S / \emptyset / N]$ will start at the price of 0 . And finally at a price higher than $\beta$, it can not manage to make any sales.

Notice that there is a unique second-stage equilibrium market structure for each firststage price $p$. Specifically, in the price range where the network might form, it is only the maximum network and not the critical-mass network that exists. This is due to the fact that $\tau>\sigma$. The implication is that coordination failure never occurs. In the partially-served market $[S / \emptyset / N]$, this is caused by the parameter setting of Assumption $2, \gamma>\sigma$. Since the firm is not directly involved at the digital end of the market (where $x$ is close to 1 ), the fact that there are fanatic P2P users joining even if no one else joins helps eliminate the possibility of a potential coordination failure. On the other hand, in the fully-served market $[S / N]$, the lack of coordination failure is caused by the parameter setting within this scenario, $\tau>\sigma$. When the degree of taste heterogeneity is high relative to the cost factor of downloading and the market is still fully served, it must be that the network is inherently so strong that the required size of a critical-mass network is extremely low (indeed negative) and therefore always fulfilled.

The existence of the partially-served, multi-platformed market structure $[S / \emptyset / N]$ is also caused by the parameter setting $\tau>\sigma$. the consumers are sufficiently differentiated such that a section of the market in the middle can be left unserved in equilibrium. Furthermore, the fact that $\gamma>\sigma$ leads to the direct result that the digital end of the market is always served, leaving the market structure $[S / \emptyset]$ an impossibility. ${ }^{5}$

In order to determine which price the firm will actually choose in stage one, one needs to investigate the profit levels of these different market structures in stage one. This will be dealt with in the next section, and we continue this section with analyzing the possible equilibrium market structures that can result from the firm's pricing in the other two scenarios.

Proposition 2. Given the price $p$ set in the first stage by the firm, all second-stage equilibrium market structures in the medium heterogeneity scenario $(\gamma>\tau>\sigma)$ are as depicted in Figure 3.

$$
\begin{aligned}
& {[S]} \\
& {[S / N]_{m . n .} .} \\
& {[N]_{m . n .} .}
\end{aligned}
$$

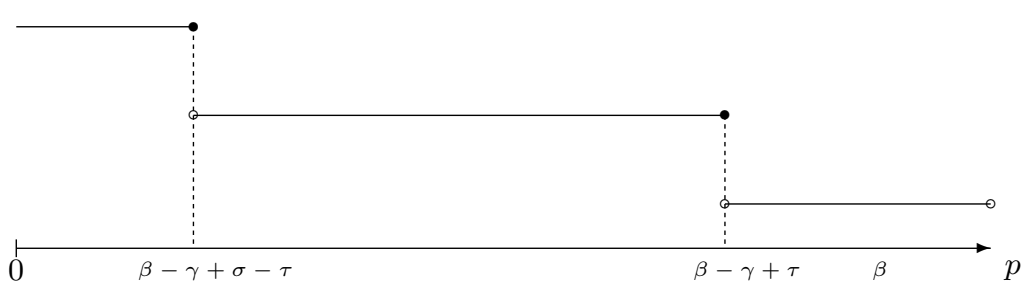

Figure 3: Medium heterogeneity scenario

Similarly to Proposition 1, there is a unique second-stage equilibrium market structure for each first-stage price $p$. Notice again in particular the lack of critical-mass network and

\footnotetext{
${ }^{5}$ These results compare nicely with those from Herings et al. (2008).
} 
coordination failure in network forming. This proposition also bares some other similarities with Proposition 1.

The firm is still able to serve the whole market here if it charges a price $p \leq \beta-\gamma+\sigma-\tau$. Again, if $\beta-\gamma+\sigma-\tau<0$, market structure $[S / N]$ will start at the price of 0 . The resulting market structure is then single-platformed fully-served market $[S]$. With a price any higher than that, a network will form and for prices $p \leq \beta-\gamma+\tau$ the resulting market structure will be $[S / N]$. Notice that, unlike in the high heterogeneity scenario (Proposition 1), the market structure $[S / \emptyset / N]$ does not exist in this scenario. This is the case because the consumers are not sufficiently differentiated for the firm to act like a local monopoly and partially serve the market. In other words, the firm is not able to ignore the network and act as a monopoly due to the lower level of taste heterogeneity $\tau$ in this scenario.

For all prices $p>\beta-\gamma+\tau$ the firm fails to make any sales and the market will be fully served by the network, hence the market structure $[N]$. Notice that this price is lower than $\beta$, which is the lower-bound price of the zero-sales market structure $[\emptyset / N]$ in Proposition 1 . This reflects the fact that, comparing to the high heterogeneity scenario, the firm is now facing less differentiated consumers and hence more direct competition from the network. It now has less freedom in price setting since it will lose all its consumers at an even lower price, comparing to the high heterogeneity scenario.

Proposition 3. Given the price $p$ set in the first stage by the firm, all second-stage equilibrium market structures in the low heterogeneity scenario $(\gamma>\sigma>\tau)$ are as depicted in Figure 4.

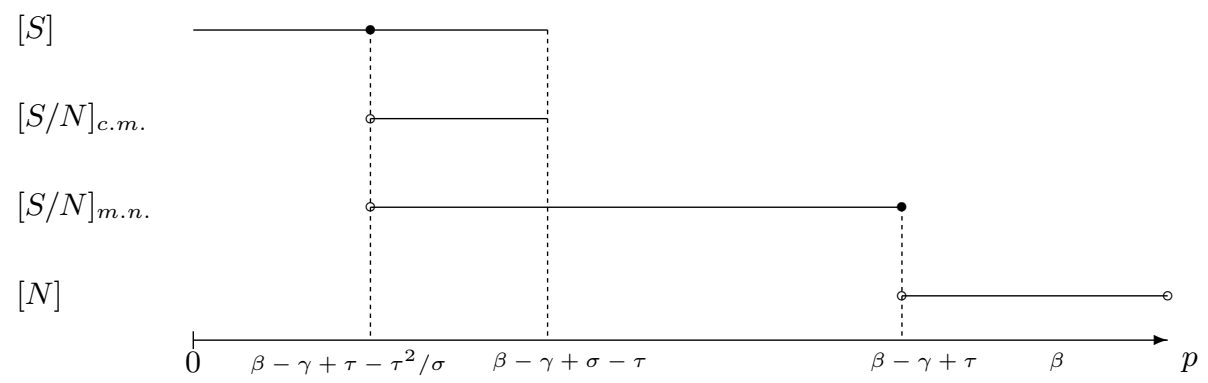

Figure 4: Low heterogeneity scenario.

The first striking difference in this proposition is that there are multiple equilibria. Secondly, unlike in the two previous propositions, we find a critical-mass network equilibrium (see Appendices for the proof). This implies that it is now possible to have a coordination failure in network forming, specifically within the price range $\left[\beta-\gamma+\tau-\tau^{2} / \sigma, \beta-\gamma+\sigma-\tau\right]$. When the firm charges a price in this range, it is possible that the consumers are able to form a network if everyone expects this to be the case, or fail to do so if everyone believes a network will not form. 
The upper-bound price of $[S / N]_{m . n .}$ is $\beta-\gamma+\tau$, as in Proposition 2. However, recall that in Proposition 1 and 2, where $\tau>\sigma$, the upper-bound price for $[S]$ and the lower-bound

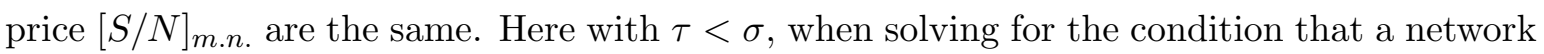
must have the size smaller than 1 , the resulting inequality gives a smaller lower-bound price for the market structures $[S / N]_{c . m}$. and $[S / N]_{m . n}$. than the upper-bound price for the market structure $[S]$. Therefore, there is a price range where these two market structures overlap, namely, when $\beta-\gamma+\tau-\tau^{2} / \sigma<p<\beta-\gamma+\sigma-\tau$. This is also the price range where the critical-mass network exists. A crucial point here is that the firm's profit levels are not continuous when switching form $[S]$ to $[S / N]$ at the price $\beta-\gamma+\tau-\tau^{2} / \sigma$. The firm can still fully serve the market alone if it charges a price no higher than $\beta-\gamma+\tau-\tau^{2} / \sigma$, provided that it is positive.

Note that it must hold that $\beta-\gamma+\tau-\tau^{2} / \sigma>0$, otherwise all three market structures $[S],[S / N]_{c . m}$. and $[S / N]_{m . n}$. start at the price of 0 , and the section in the graph where $[S]$ is the unique market structure will not exist. Moreover, for the section in the graph where $[S / N]_{m . n}$. is the unique the market structure in the price range $[\beta-\gamma+\sigma-\tau, \beta-\gamma+\tau]$, it must hold that $\beta-\gamma+\tau>\beta-\gamma+\sigma-\tau$, which means $\sigma<2 \tau$. Otherwise, all three market structures $[S],[S / N]_{c . m}$. and $[S / N]_{m . n}$. will end at the price of $p=\beta-\gamma+\tau$, where the market structure $[N]$ starts.

\section{Firm's decision}

The firm sets a profit maximizing price $p$ in stage one, taking into account the possible realized equilibrium market structure in stage two. Depending on the different scenarios in stage two we discussed in the previous section, the optimum price $p$ can vary in stage one.

In this section, we investigate subgame-perfect equilibrium prices and market outcomes for each of the scenarios analyzed in the previous section. In the next section, we will then summarize and regroup the findings with respect to the equilibrium market structures in order to provide more economic insights and intuitions.

In the low heterogeneity scenario $(\gamma>\sigma>\tau)$, the possible equilibrium market structures in stage two involve multiple equilibria. To deal with the multiplicity of subgame-perfect equilibria, we follow the convention by supposing that, once the price is known, consumers coordinate on the equilibrium continuation that they prefer, which is the one with the largest network size. ${ }^{6}$

Similar to Herings et al. (2008), we adopt the approach where the second-stage equilibrium market structure that corresponds to the firm-worst response of the consumers, given the first-stage price $p$, is captured by a lower envelop. This is depicted in Figure 5 below. The lower envelop is the collection of the three solid lines. Notice that within the price range $\left[\beta-\gamma+\tau-\frac{\tau^{2}}{\sigma}, \beta-\gamma+\sigma-\tau\right]$, where multiple second-stage equilibria occur, only the one

\footnotetext{
${ }^{6}$ See, for instance, Katz and Shapiro (1986) or Fudenberg and Tirole (2000).
} 
with the lowest profit for the firm, i.e. $[S / N]_{m . n .}$, is on the lower envelop. Here, we do not try to produce a full characterization of all subgame-perfect equilibria. ${ }^{7}$ We will concentrate instead on the equilibrium continuation that consumers prefer the most, i.e. the one with the largest network, and assume that they coordinate on this equilibrium continuation after the price is known. This in fact coincides with the firm-worst situation represented by the lower envelop.

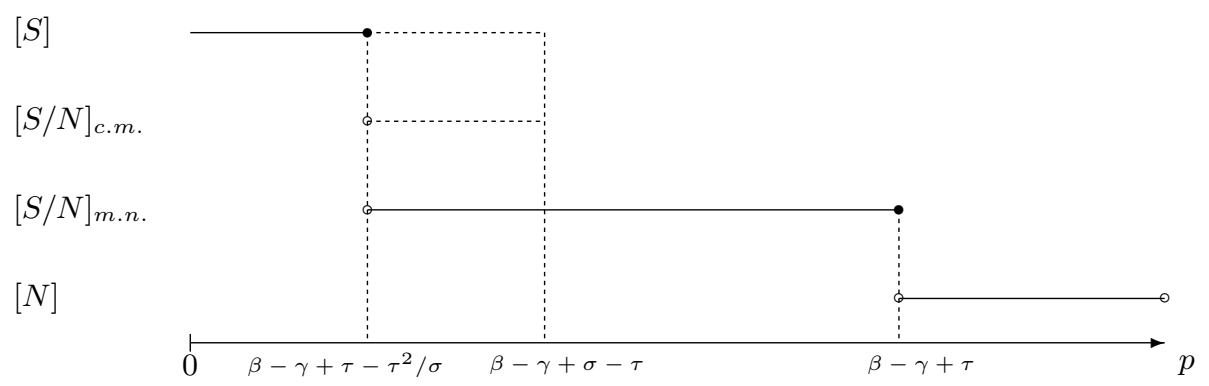

Figure 5: The lower envelop of the low heterogeneity scenario.

For the convenience in notation, we define $\delta \equiv 4 \tau^{2}-3 \sigma(\beta-\gamma+\tau)$. Roughly speaking, $\delta$ represents the attractiveness of the network relative to the firm. Indeed, $\delta$ is increasing in $\gamma-\beta$ and decreasing in $\sigma$.

Furthermore, we use $\Pi_{[\cdot]}^{*}$ to denote the maximum profit of any market structure [.] as depicted in Table 1.

Proposition 4. In the high heterogeneity scenario $(\tau>\gamma>\sigma)$, there are parameter settings such that the following four market outcomes and their associated price and profit levels can be supported by a subgame-perfect equilibrium.

1. The firm serves the entire market by charging a price $p=\beta-\gamma+\sigma-\tau$, and makes a profit level of $\Pi_{[S]}^{*}=\beta-\gamma+\sigma-\tau$.

2. The firm competes directly with the network by charging a price $p=\frac{2}{9 \sigma}(2 \tau-\sqrt{\delta})(\tau+\sqrt{\delta})$ and earns a profit of $\Pi_{[S / N]}^{*}=\frac{2}{27 \sigma^{2}}(2 \tau-\sqrt{\delta})^{2}(\tau+\sqrt{\delta})$.

3. The firm serves precisely up to the last consumer who does not download from the network by charging a price $p=\beta-\frac{\tau}{2 \sigma}\left(\tau-\sqrt{\tau^{2}-4 \sigma(\tau-\gamma)}\right)$, and earns a profit of $\Pi_{[S / N]-\text { boundary }}=\left(\tau-\sqrt{4 \gamma \sigma-4 \sigma \tau+\tau^{2}}\right)\left(2 \beta \sigma-\tau^{2}+\tau \sqrt{4 \gamma \sigma-4 \sigma \tau+\tau^{2}}\right) / 4 \sigma^{2}$.

4. The firm acts as a local monopoly and charges the price $p=\frac{\beta}{2}$ and earns a profit of $\Pi_{[S / \emptyset / N]}^{*}=\frac{\beta^{2}}{4 \tau}$.

Proof.

It is helpful to look back at Figure 2. First of all, any price $p>\beta$ can not be profit-maximizing,

\footnotetext{
${ }^{7}$ For a vigorous derivation of such a full characterization, see Herings et al. (2008).
} 
since under the market structure of $[\emptyset / N]$ the firm's sales quantity and profit are invariably 0 , and the firm can always do better by charging a price that yields a positive sales quantity. We therefore conclude that this market structure can not be supported by any subgame-perfect equilibrium.

Secondly, any price $p<\beta-\gamma+\sigma-\tau$ can not be profit-maximizing, since the resulting sales quantity is always 1 (the entire market), and the profit function is continuous and monotonic in price. This makes $p=\beta-\gamma+\sigma-\tau$ strictly more profitable than any lower price. Therefore, the highest profit under the market structure $[S]$ corresponds to this price $p=\beta-\gamma+\sigma-\tau$. Moreover, the profit levels are continuous at this price where the market structure switches from $[S]$ to $[S / N]$. The first derivative of the profit function with respect to price under the market structure $[S / N]$ is not strictly positive under the condition $\beta-\gamma \geq 3(\tau-\sigma)$, which implies that the profit at this price can be a global maximum profit and hence may be supported by a subgame-perfect equilibrium. However, when $\beta-\gamma<3(\tau-\sigma)$, the profit levels at this price is both continuous and monotonic, in which case the profit at this price can not be a global maximum.

The profit levels under the market structure of $[S / N]$, can be non-monotonic with a local maximum value of $\Pi_{[S / N]}^{*}=\frac{2}{27 \sigma^{2}}(2 \tau-\sqrt{\delta})^{2}(\tau+\sqrt{\delta})$, at the price of $p_{[S / N]}^{*}=\frac{2}{9 \sigma}(2 \tau-\sqrt{\delta})(\tau+$ $\sqrt{\delta}$ ), under the condition that $\delta \geq 0$. This profit level can be a global maximum, hence may be supported by a subgame-perfect equilibrium.

However, if $\delta<0$, the maximum profit of $[S / N]$ is achieved at the upper boundary price $p=\beta-\frac{\tau}{2 \sigma}\left(\tau-\sqrt{\tau^{2}-4 \sigma(\tau-\gamma)}\right)$, yielding a profit level of $\Pi_{[S / N] \text {-boundary }}=(\tau-$ $\left.\sqrt{4 \gamma \sigma-4 \sigma \tau+\tau^{2}}\right)\left(2 \beta \sigma-\tau^{2}+\tau \sqrt{4 \gamma \sigma-4 \sigma \tau+\tau^{2}}\right) / 4 \sigma^{2}$. This can be supported by a subgameperfect equilibrium only if the profit under the market structure $[S / \emptyset / N]$ at this price is decreasing and the profit function is strictly monotonic across its price range until its upperbound price $p=\beta$.

Finally, the profit levels under the market structure of $[S / \emptyset / N]$, also can be non-monotonic. Under this market structure, the firm is acting as a monopoly and does not compete directly with the network. The consumer $x$ who is indifferent from buying the physical product in the store and not buying has utility level $\beta-\tau x-p=0$, which implies $x=(\beta-p) / \tau$. Taking this as a demand function and the firm optimizes its price at $p_{[S / \emptyset / N]}^{*}=\beta / 2$, resulting a profit level of $\Pi_{[S / \emptyset / N]}^{*}=\beta^{2} / 4 \tau$. For this interior solution to be the maximum profit in this price range, two conditions have to be met. The first one is that $x<1$, which implies that $\beta<2 \tau$ must hold. The second one requires that the price $p_{[S / \emptyset / N]}^{*}=\beta / 2$ to be in the price range of $[S / \emptyset / N]$, in particular, $\beta<\tau^{2}-\tau \sqrt{\tau^{2}-4 \sigma(\tau-\gamma)}$. This maximum profit can be a global maximum, hence may be supported by a subgame-perfect equilibrium.

In order to demonstrate the existence of parameter settings that qualify the above four numbered items to be subgame-perfect equilibrium outcomes, we provide for each of them a set of example parameters in Table 2. Each of the four columns of the table presents a set 
of parameter values that yield the corresponding profit level as the global maximum, in the order of the four bullets points described in Proposition 4.

\begin{tabular}{l|cccc}
\hline & \multicolumn{4}{|c}{ Equilibrium outcomes } \\
Parameters & $\Pi_{[S]}^{*}$ & $\Pi_{[S / N]}^{*}$ & $\Pi_{[S / N]}-$ boundary & $\Pi_{[S / \emptyset / N]}^{*}$ \\
\hline$\beta$ & 19 & 12 & 12 & 10 \\
$\gamma$ & 9 & 8 & 8 & 9 \\
$\sigma$ & 8 & 7 & 7 & 8 \\
$\tau$ & 10 & 10 & 12 & 14 \\
\hline
\end{tabular}

Table 2: Examples of parameter values for Proposition 4 (high heterogeneity scenario)

The relevant profit functions given these four sets of parameter values are as depicted in Figure 6 . The four pictures represent the four sets of parameter values depicted in Table 2 respectively. In each picture, the firm's profit level is depicted as a function of its price. Different line styles represent different market structures, which are labeled by the profit legends $\left(\Pi_{[S]}, \Pi_{[S / N]}, \ldots\right)$. The global maximum profits in these four pictures are achieved under the market structures indicated by the market legends at the bottom of each picture $([S],[S / N], \ldots)$, as described in Proposition 4.
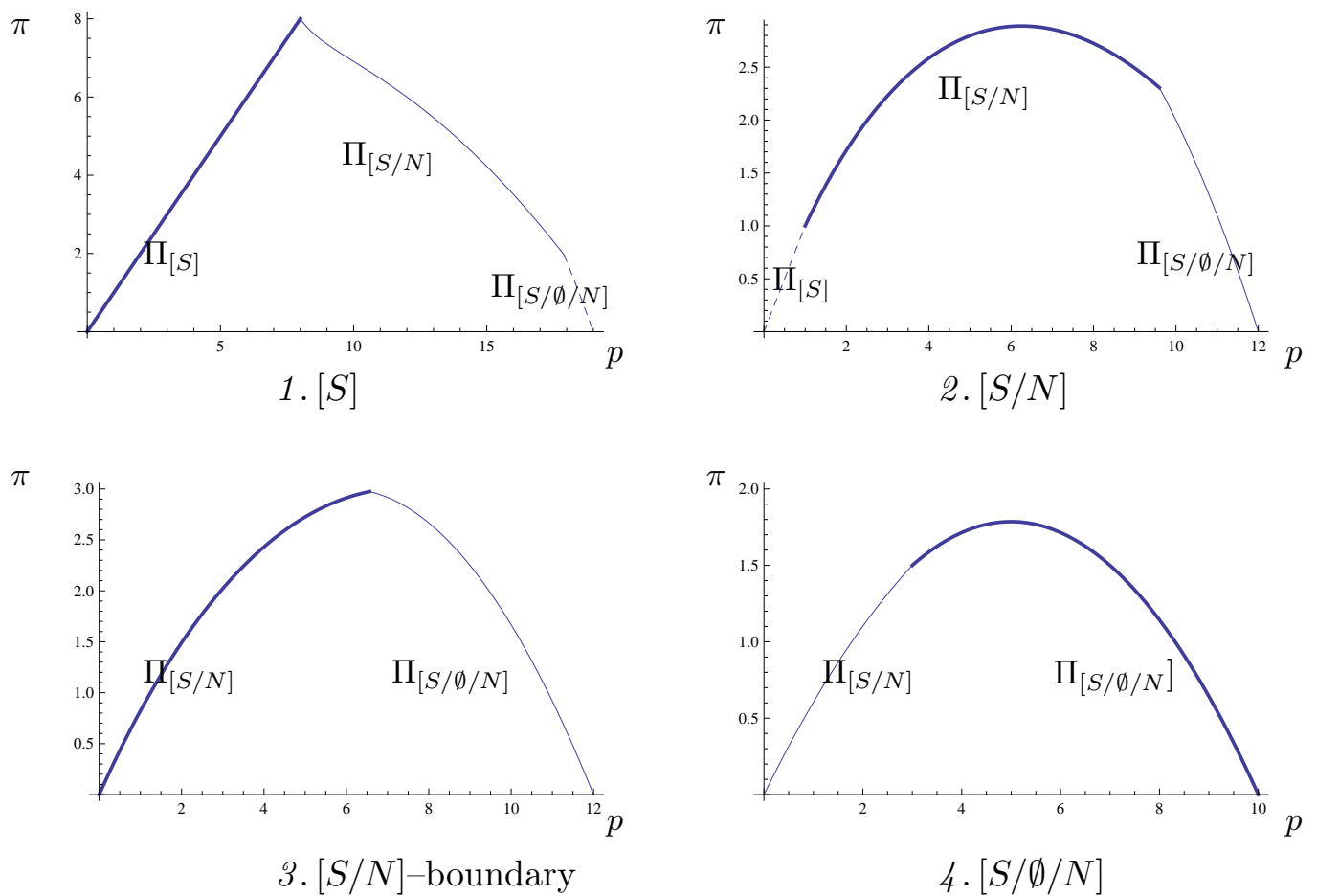

Figure 6: Profit functions and equilibrium outcomes (high heterogeneity scenario)

We only plot the profit levels in the relevant price range, namely, $p \in[0, \beta]$. Notice the continuity of profit levels in all four pictures while rising and falling with prices across different 
market structures.

Proposition 5. In the medium heterogeneity scenario $(\gamma>\tau>\sigma)$, there are parameter settings such that the following two market outcomes and their associated price and profit levels can be supported by a subgame-perfect equilibrium.

1. The firm serves the entire market by charging a price $p=\beta-\gamma+\sigma-\tau$, and makes a profit level of $\Pi_{[S]}^{*}=\beta-\gamma+\sigma-\tau$.

2. The firm competes directly with the network by charging a price $p=\frac{2}{9 \sigma}(2 \tau-\sqrt{\delta})(\tau+\sqrt{\delta})$ and earns a profit of $\Pi_{[S / N]}^{*}=\frac{2}{27 \sigma^{2}}(2 \tau-\sqrt{\delta})^{2}(\tau+\sqrt{\delta})$.

Proof.

In this scenario, as Figure 3 shows, there are three possible equilibrium market structures in stage two given firm's price $p$ from stage one.

Similar to Proposition 4, the maximum profit under the market structure $[S]$ is achieved at the boundary price $p=\beta-\gamma+\sigma-\tau$. Under the condition that $\beta-\gamma \geq 3(\tau-\sigma)$, the first derivative of the profit function $\Pi_{[S / N]}$ at this price is non positive, which implies that this profit level can be a global maximum, hence may be supported by a subgame-perfect equilibrium.

If $\beta-\gamma<3(\tau-\sigma)$, the profit levels under the market structure $[S]$ are strictly dominated by at least one profit level under the market structure $[S / N]$. In this case, The profit function $\Pi_{[S / N]}$ reaches a local maximum $\Pi_{[S / N]}^{*}=\frac{2}{27 \sigma^{2}}(2 \tau-\sqrt{\delta})^{2}(\tau+\sqrt{\delta})$, before subsequently decreasing until reaching 0 at the upper-bound price $p=\beta-\gamma+\tau$.

Finally, the market structure $[N]$ with the firm having zero profit can not be sustained by any subgame-perfect equilibrium either, since the firm can always do better by charging a lower price in order to have at least some sales.

Again we demonstrate the existence of parameter settings that qualify the above two numbered items to be subgame-perfect equilibrium outcomes by providing for each of them a set of example parameters in Table 3. Each of the two columns of Table 3 presents a set of parameter values that yield the corresponding profit level as the global maximum, in the order of the two numbered items described in Proposition 5.

\begin{tabular}{l|cc}
\hline & \multicolumn{2}{|c}{ Equilibrium outcomes } \\
Parameters & $\Pi_{[S]}^{*}$ & $\Pi_{[S / N]}^{*}$ \\
\hline$\beta$ & 16 & 13 \\
$\gamma$ & 11 & 11 \\
$\sigma$ & 9 & 9 \\
$\tau$ & 10 & 10 \\
\hline
\end{tabular}

Table 3: Examples of parameter values for Proposition 5 (medium heterogeneity scenario) 
The relevant profit functions given these two sets of parameter values are as depicted in Figure 7. The two pictures represent the two sets of parameter values depicted in Table 3 respectively. Notice once again the continuity in profit levels across market structures.

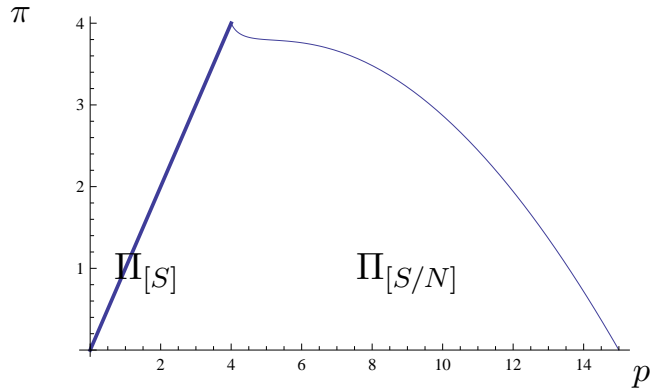

1. $[S]$

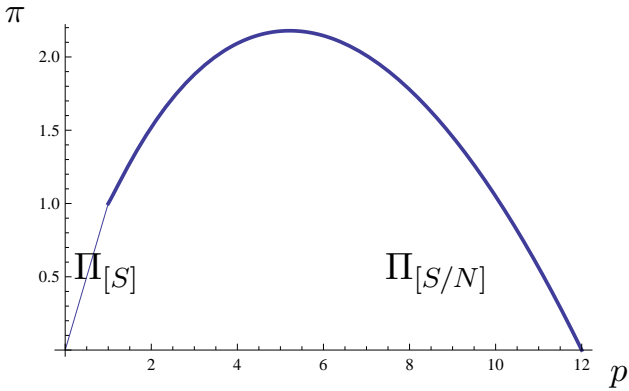

2. $[S / N]$

Figure 7: Profit functions and equilibrium outcomes (medium heterogeneity scenario)

Proposition 6. In the low heterogeneity scenario $(\gamma>\sigma>\tau)$, there are parameter settings such that the following two market outcomes and their associated price and profit levels can be supported by a subgame-perfect equilibrium.

1. The firm sets a network-deterring price $p=\beta-\gamma+\tau-\frac{\tau^{2}}{\sigma}$ and serves the entire market alone, making a profit of $\Pi_{[S]}^{*}=\beta-\gamma+\tau-\frac{\tau^{2}}{\sigma}$.

2. The firm competes directly with the network by charging a price $p=\frac{2}{9 \sigma}(2 \tau-\sqrt{\delta})(\tau+\sqrt{\delta})$ and earns a profit of $\Pi_{[S / N]}^{*}=\frac{2}{27 \sigma^{2}}(2 \tau-\sqrt{\delta})^{2}(\tau+\sqrt{\delta})$.

Proof.

It is helpful to look back at the lower envelop in Figure 5 at the beginning of the section.

First of all, as the previous propositions, the last part of the lower envelop representing the second stage equilibrium $[N]$ clearly can not yield any possible subgame-perfect equilibrium outcome, because the firm is making zero profit. However, the other two sections, namely $[S]$ and $[S / N]_{m . n .}$, can.

The highest profit level on the $[S]$ section of the lower envelop is achieved at the right-hand extreme of the line at the price of $p_{[S]}^{*}=\beta-\gamma+\tau-\frac{\tau^{2}}{\sigma}$, under the condition that it is positive. Since sales quantity is 1 in a fully-served market, this yields a profit of $\Pi_{[S]}^{*}=\beta-\gamma+\tau-\frac{\tau^{2}}{\sigma}$, which can be a global maximum profit and hence may be supported by a subgame-perfect equilibrium.

The profit function on the section $[S / N]_{m . n}$. is similar to the previous two propositions. However, observe the discontinuity of the profit levels from the market structure $[S]$ to $[S / N]_{m . n}$. at the price level $p=\beta-\gamma+\tau-\frac{\tau^{2}}{\sigma}$. In fact, the profit level at this price under the market structure $[S / N]_{m . n}$. is exactly a fraction $\frac{\tau}{\sigma}$ of the profit under the market structure of $[S]$. In other words, a small increase in price triggers a network of the size $1-\frac{\tau}{\sigma}$ 
to form and significantly hurts the sales quantity of the firm. The profit function $\Pi_{[S / N]_{m . n} \text {. }}$ does increase in price though, until reaching its maximum $\Pi_{[S / N]_{m . n} .}^{*}=\frac{2}{27 \sigma^{2}}(2 \tau-\sqrt{\delta})^{2}(\tau+\sqrt{\delta})$ at the price $\Pi_{[S / N]}^{*}=\frac{2}{27 \sigma^{2}}(2 \tau-\sqrt{\delta})^{2}(\tau+\sqrt{\delta})$, before decreasing again all the way to zero, under the condition $\delta \geq 0$. This can be a global maximum profit and hence may be supported by a subgame-perfect equilibrium.

Note that the maximum profit under the market structure $[S / N]$ is achieved at its lowerbound price if $\delta<0$, which implies $\beta-\gamma+\tau-\frac{4 \tau^{2}}{3 \sigma}>0$. This fulfills the condition that $\beta-\gamma+\tau-\frac{\tau^{2}}{\sigma}>0$. In that case the firm will indeed charge a price at $p_{[S]}^{*}=\beta-\gamma+\tau-\frac{\tau^{2}}{\sigma}$ to deter the network.

As with the previous propositions, we demonstrate the existence of parameter settings that qualify the above two numbered items to be subgame-perfect equilibrium outcomes by providing for each of them a set of example parameters in Table 4.

\begin{tabular}{l|cc}
\hline & \multicolumn{2}{|c}{ Equilibrium outcomes } \\
Parameters & $\Pi_{[S]}^{*}$ & $\Pi_{[S / N]}^{*}$ \\
\hline$\beta$ & 14 & 12 \\
$\gamma$ & 13 & 11 \\
$\sigma$ & 12 & 9.5 \\
$\tau$ & 10 & 9 \\
\hline
\end{tabular}

Table 4: Examples of parameter values for Proposition 6 (low heterogeneity scenario)

The relevant profit functions given these two sets of parameter values are as depicted in Figure 8. The two pictures represent the two sets of parameter values depicted in Table 4 respectively. Notice this time the discontinuity in profit levels across market structures.

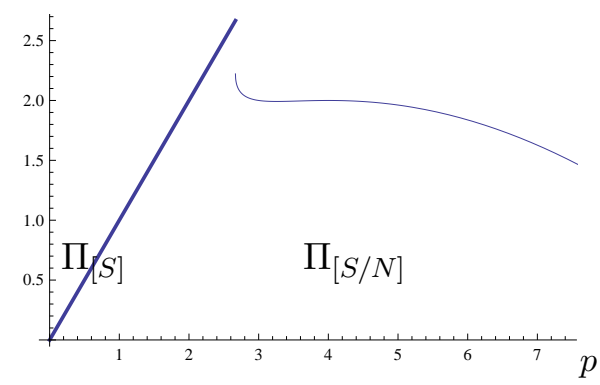

1. $[S]$

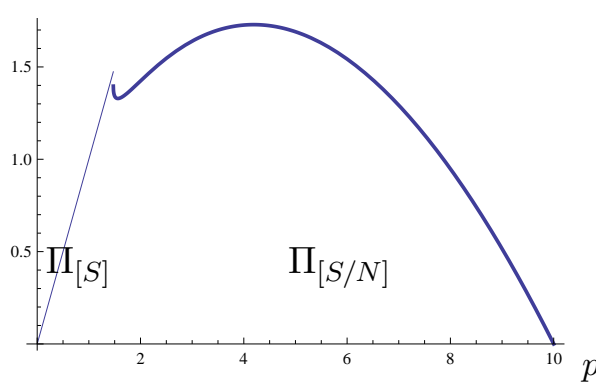

2. $[S / N]$

Figure 8: Profit functions and equilibrium outcomes (low heterogeneity scenario) 


\section{$5 \quad$ Equilibrium market structures}

We summarize the analysis of the scenarios above by means of the following four corollaries, in which we show the optimal prices of the firm and the resulting market structures that are supported by a subgame-perfect Nash equilibrium.

Corollary 1. Partially-served local monopoly.

When $\tau$ is sufficiently large $(\tau>\gamma>\sigma)$, the firm can act as a monopoly while ignoring the P2P network by charging a price $p^{*}=\frac{\beta}{2}$ and making a profit of $\Pi_{[S / \emptyset / N]}^{*}=\frac{\beta^{2}}{4 \tau}$. The outcome is a multi-platformed partially-served market - $[S / \emptyset / N]$.

This result is stemming from the high heterogeneity scenario. When the taste heterogeneity $\tau$ is high enough, the consumers are sufficiently differentiated so that in equilibrium the firm can safely disregard the network at the other end of the market (those who prefer the digital form will therefore download) and charge its monopolistic price to its "own" consumers. The two platforms will, therefore, co-exist and not compete; and the market will be partially served.

Corollary 1, in combination with Herings et al. (2008), shows that $\tau>\gamma$ is a necessary condition for a multi-platformed partially-served market. One could interpret $\tau>\gamma$ as the situation that tastes differ so much across consumers that some consumers would not have the illegal version even if downloading it costs zero time and effort.

Corollary 2. Fully-served monopoly.

When $\tau$ is not too small, the firm can serve the entire market at the profit-maximizing price level $p^{*}=\beta-\gamma+\sigma-\tau$ and a profit level of $\Pi_{[S]}^{*}=\beta-\gamma+\sigma-\tau$, and the outcome is a single-platformed fully-served market $-[S]$.

This result is stemming from the high and medium heterogeneity cases. The firm's natural profit maximizing sales quantity is the entire market. This is chiefly due to the fact that the basic utility of the physical product is so high that the taste heterogeneity cost becomes insignificant in comparison. Although this is theoretically possible, in reality the taste heterogeneity is probably high enough that in equilibrium not everyone will be naturally served by the monopoly. Therefore, we will not dig too much deeper in the analysis of this market outcome.

Corollary 3. Network deterrence.

When $\tau$ is very small $(\gamma>\sigma>\tau)$, the firm can serve the entire market and thereby deter the entry of the network by charging a price of $p^{*}=\beta-\gamma+\tau-\frac{\tau^{2}}{\sigma}$, making a profit of $\Pi_{[S]}^{*}=\beta-\gamma+\tau-\frac{\tau^{2}}{\sigma}$, and the outcome is a single-platformed fully-served market $-[S]$.

This result is stemming from the low heterogeneity scenario. Note that opposite to Corollary 1 , where $\tau$ is the biggest, in Corollary $3, \tau$ is the smallest. This proposition demonstrates 
that under certain parameter conditions, there is a possibility for the firm to deter the network from forming by an aggressive pricing strategy. Observe that this is only possible when the taste heterogeneity is very small. Since consumers never fail to coordinate on forming a network (owing to Assumption 2), the only way for the firm to deter the network is to serve the whole market itself, even if, by doing so, it can no longer charge a monopoly price. Indeed, under these conditions, if the firm ignores the network and charges the normal monopoly price, the network would form and reach its maximum equilibrium size and cause some serious damage on the firm's sales. The firm is, therefore, better off deviating from the monopoly price and setting a network-deterring price.

\section{Corollary 4. Market sharing}

When $\tau$ is very large, the firm serves precisely up to the last consumer who does not download from the network by charging a price $p=\beta-\frac{\tau}{2 \sigma}\left(\tau-\sqrt{\tau^{2}-4 \sigma(\tau-\gamma)}\right)$, and earns a profit of $\Pi_{[S / N]-\text { boundary }}=\left(\tau-\sqrt{4 \gamma \sigma-4 \sigma \tau+\tau^{2}}\right)\left(2 \beta \sigma-\tau^{2}+\tau \sqrt{4 \gamma \sigma-4 \sigma \tau+\tau^{2}}\right) / 4 \sigma^{2}$. The market outcome is a fully-served multi-platformed market without the two platforms competing with each other $-[S / N]-$ boundary.

This is a rather special market outcome, where the firm and the network together "share" the whole market without actually competing with each other. It relies on the fact that the taste heterogeneity is sufficiently large such that the firm does not have to fight hard with the network for the indifferent consumer; but at the same time not so large such that the firm enjoys being a local monopoly as in Corollary 1 . Note that the firm is actually pricing higher than its monopolistic price due to the presence of the network. For at the monopolistic price, the firm would face competition from the network and thereby unable to achieve monopolistic quantity. Thus, the firm sets the price at exactly the level so as to leave the network alone and served the entire residual demand of the market.

Corollary 5. Network accommodation.

Regardless of the value of $\tau$, there are parameter settings where the firm competes directly with the P2P network by charging a price $p^{*}=\frac{2}{9 \sigma}(2 \tau-\sqrt{\delta})(\tau+\sqrt{\delta})$, making a profit of $\Pi_{[S / N]}^{*}=\frac{2}{27 \sigma^{2}}(2 \tau-\sqrt{\delta})^{2}(\tau+\sqrt{\delta})$, and the market outcome is a multi-platformed fully-served market $-[S / N]$.

This corollary captures all the rest of the parameter space which is not covered in Corollary 1, 2, 3 and 4. It is essentially the relation $\gamma>\sigma$ that makes the network readily available, and that makes network accommodation (co-existence) a prominent phenomenon.

The corollaries summarize the essential findings of this paper and tells a rather compelling story. When consumers view the two versions as very different ( $\tau$ being large), the firm may enjoy the luxury of not having to deal with the network since its own clientele is by no means affected. For this to happen, the basic utility of the illegal version $\gamma$ should be relatively small and/or the generic cost factor of downloading $\sigma$ should be relatively high. In this case, 
aggressive price competition is not called for. When the taste differences are less pronounced, however, price competition with the network cannot be avoided. The firm has to accommodate the network, and they fight with each other for the marginal consumer. Together they serve the entire market. The situation changes even further when the taste heterogeneity reaches a very low level. In that case, consumers no longer have high disutility for consuming the least preferred version, and the firm may have a chance to price aggressively in order to grab hold of the whole market and deter the network from forming.

Furthermore, $\delta=4 \tau^{2}-3 \sigma(\beta-\gamma+\tau)$ plays an important role in determining market outcomes. Recall that $\delta$ corresponds to the attractiveness of the network relative to the firm. For the firm to ignore the existence of the network, $\delta$ has to be relatively small. This requires, among other things, $\gamma$ to be relatively small and $\tau$ to be relatively large. It is consistent with what is required by Corollary 1. Alternatively, for the firm to be able to deter the entry of the network, $\delta$ has to be small as well. But Corollary 3 requires $\gamma$ to be large and $\tau$ to be small, which makes $\delta$ relatively large. This implies that $\sigma$ and $\beta$ have to be relatively large for $\delta$ to be small. Intuitively, if consumers do not care too much about the difference between versions, the firm can only deter the network if its product is much more superior in quality and the generic factor of downloading is relatively large. This may in reality prove hard to achieve.

\section{Comparative statics}

The previous section shows how taste heterogeneity $\tau$ can affect the possible equilibrium outcome in the market. It is essentially a comparative static analysis across equilibrium market structures. It may be interesting also to look at comparative statics of all the main parameters of the model in given equilibrium market structures. In Herings et al. (2008), the comparative statics of many market structures have been extensively studied, including the single-platformed as well as the multi-platformed fully-served markets. The main findings there are the following. The larger the generic cost factor of downloading is, the less likely a P2P network will form, and the higher the firm's profit will be. Once the market exhibits co-existence of the two platforms, the smaller the cost factor is, the higher the price set by the firm. Despite this pricing behavior, the firm's profit unambiguously declines as the cost factor decreases. In the partially-served monopolistic market with network-deterring pricing and the fully-served market with multi-platform co-existence, the total welfare decreases in the cost factor. For a comprehensive overview of this, we refer to that paper.

Due to the different parameter settings in this paper - smaller $\sigma$ and less restrictions on $\tau$ - two additional market outcomes can be supported by a subgame-perfect equilibrium. One is a naturally fully-served monopoly market $[S]$, and the other a multi-platformed partiallyserved local monopoly market $[S / \emptyset / N]$. We focus on the latter more interesting one and look at its comparative statics. Here the firm is ignoring the network all together. It charges a 
monopoly price $p^{*}$, serving a market the size of $s^{*}$, and making a profit of $\Pi^{*}$. The network lives at the other end of the market with a size of $n^{*} .{ }^{8}$ We will also look at the effects on consumers' surplus and the total welfare, denoted by $C S^{*}$ and $W^{*}$, respectively.

Proposition 7. The signs of the first order partial derivatives of the equilibrium values with respect to the parameters in the case of the monopoly market are as shown in Table 5.

\begin{tabular}{lcccc}
\hline & $\beta$ & $\gamma$ & $\tau$ & $\sigma$ \\
\hline$p^{*}$ & + & 0 & 0 & 0 \\
$s^{*}$ & + & 0 & - & 0 \\
$n^{*}$ & 0 & + & - & - \\
$\Pi^{*}$ & + & 0 & - & 0 \\
$C S^{*}$ & + & + & - & - \\
$W^{*}$ & + & + & - & - \\
\hline
\end{tabular}

Table 5: Multi-platformed partially-served (local monopoly) market.

Proof.

The comparative statics of all parameters on $p^{*}=\frac{\beta}{2}, s^{*}=\frac{\beta}{2 \tau}$, and $\Pi^{*}=\frac{\beta^{2}}{4 \tau}$ are straight forward. The equilibrium network size $n^{*}=1-\frac{1}{2 \sigma}\left(\tau-\sqrt{\tau^{2}-4 \sigma(\tau-\gamma)}\right)$ does not depend on $\beta$, and it is easy to see that it increases in $\gamma$. The first derivative of $n^{*}$ with respect to $\tau$ and $\sigma$ can be shown to be both negative.

The consumers' surplus can be divided into two components: the surplus for the buyers and the surplus for the downloaders, which we denote by $C S_{S}^{*}$ and $C S_{N}^{*}$, respectively. The expression for the former $C S_{S}^{*}=\frac{\beta^{2}}{8 \tau}$. It is clear that the signs of its derivatives with respective to $\beta, \gamma, \tau$ and $\sigma$ are $+, 0,-$ and 0 . The expression of $C S_{N}^{*}$ is slightly more involved, but we know that it is the integral of the utility functions of the downloaders, which is $\gamma-\tau(1-x)-\sigma(1-n)^{2}$, with respect to $x$. It is clear that $\tau$ and $\sigma$ both have negative effects on each downloader's utility. Moreover, we know that they also have negative effects on the network size $n$. This shows that the total effects of $\tau$ and $\sigma$ must be negative on downloaders' surplus $C S_{N}^{*}$. Combined with the effects on the buyers' surplus $C S_{S}^{*}$, it can be seen that the effects of $\beta, \gamma, \tau$ and $\sigma$ on consumers' surplus $C S^{*}$ are,,++- and - , respectively. The effects on the total welfare are then simply the sum of the effects on consumers' surplus $C S^{*}$ and the firm's profit $\Pi^{*}$, which are unambiguous.

The intuition of the negative effect of the generic cost factor of downloading $\sigma$ on the equilibrium network size $n^{*}$ is self-evident: a higher cost factor makes the network less attractive and therefore resulting in a smaller equilibrium network. The cost factor does not have an effect on the surplus of buyers of the physical product, but does have a negative affect on that of the downloaders. This is reflected both by the direct multiplicative effect of downloading

\footnotetext{
${ }^{8}$ See Appendices for the derivations.
} 
cost and the indirect negative effect of $\sigma$ on the equilibrium network size $n^{*}$. Since $\sigma$ does not affect the firm's profit, it does not inflict further harm to the society other than crippling the downloaders.

The negative welfare effects of the taste heterogeneity $\tau$ is rather logical. Firstly, $\tau$ has a negative effect on the firm's profit $\Pi^{*}$, because for given basic utility $\beta$, a high $\tau$ leads to a lower sales quantity while the price remains constant. Thus, despite the fact that the firm has a monopoly position caused by taste heterogeneity, its profit suffers when it increases. Next, the consumers' surplus of the buyers of the physical product is negatively affected by $\tau$, not because of the price, but because of disutility of transportation cost for each consumer and the smaller equilibrium sales quantity $s^{*}$. The surplus from the downloaders is even more negatively affected by $\tau$, not only because $\tau$ represents transportation cost deducted from the utility, but also because $\tau$ decreases the equilibrium network size $n^{*}$ which is in turn reflected in a higher downloading cost (a negative utility). Taste heterogeneity in this case is undoubtedly bad for the society as a whole.

\section{Concluding remarks}

This paper adopts the model from Herings et al. (2008) and changes the parameter settings to accommodate the inherent strength of today's peer-to-peer file-sahring networks. In particular, we consider that there are always some "die-hard" P2P fans who contribute to the network's existence, and we allow the cases for relatively high level of consumer taste heterogeneity. As a result, the fight against a P2P network becomes much tougher as the consumers rarely fail to coordinate on forming a network. We find in total five equilibrium market structures, although under most conditions, the network will prevail and the firm can do nothing else than accommodating it and pricing against it to serve the entire market together. In this case, the firm's profit suffers and the total welfare benefits from the existence of P2P.

There may be some special conditions under which the firm still might have the upper hand. The firm can, for instance, find itself in the position to want to optimally serve the whole market, if the value of its product is significantly higher than that of the pirated version, and the taste heterogeneity is low. Another example is when the subjective taste heterogeneity becomes very small, coupled with a relatively high value of the physical form comparing to the digital form, the firm might be able to charge a low enough price to deter the network from forming. One practical example could be the newspaper market. The value of a particular newspaper might drop drastically when it is one-day old. Therefore the value of an original newspaper may be considerably higher than a copied version. Thus if the newspaper is priced competitively enough, it might completely prevent anyone from having any incentives to share it on the P2P networks.

Another rather special market structure is market-sharing, which happens when the taste heterogeneity is just so that the firm chooses to precisely serve the consumers who prefer 
not to download. In this situation the firm is actually pricing higher than its monopolistic price due to the presence of the network. For at the monopolistic price, the firm would face competition from the network and thereby unable to achieve the monopolistic quantity. Thus, the firm sets the price at exactly the level such that the consumer who is indifferent between downloading and not consuming will actually buy the physical form of the product from the firm. In other words, the firm lets the network form to its maximum size and serves the residual demand of the market.

The other end of spectrum is when the taste heterogeneity is huge. In this case, the products are sufficiently differentiated and the firm and the network are co-existing as local monopolies without competition. Under this multi-platformed partially-served market structure, the quality of the two versions of the product positively affect the price, the sales quantity and the profit of the firm, as well as the consumers' surplus and hence welfare as a whole. Taste heterogeneity unambiguously hurts the sales, the profits and consumers' surplus and hence total welfare, although it has no effect on the price. It is clear that it is the large taste heterogeneity that gives rise to this monopolistic market structure in the first place, so it is not surprising that it hurts welfare, although it is interesting to observe that it also hurts the firm's profit. The generic cost factor of downloading $\sigma$ decreases the total welfare because it hurts the downloading consumers but does not benefit the purchasing consumers or the firm's profit.

Finally, it is useful to stress that except for these special conditions mentioned above, the firm has no other option but to face the network and compete head-on with it in price. The resulting equilibrium market structure is the multi-platformed fully-served market where the firm and the network co-exist and compete for the marginal consumer. This is much studied in Herings et al. (2008), and most important results in that paper still hold here, except for the fact that the parameter space for this equilibrium market structure may be more pervasive in this paper.

The pervasiveness of the co-existence market structure in this paper may explain why, despite all the effort from the authorities and the publishing firms in the chasing and hunting of $\mathrm{P} 2 \mathrm{P}$ users, the results have not been hugely impressive, and internet piracy is still by and large an eminent phenomenon. Perhaps it is precisely those few, who believe in and stand by the principle of sharing and continue to provide the vital "critical mass" to the rest, that contribute to the prevalence of such file-sharing communities. And given the technology and the dispersion of the user base of such networks, it is less and less likely for anyone to technically eliminate such file sharing. It looks like the information good providers of the $21 \mathrm{st}$ century really have their work cut out for them. 


\section{Discussion}

It might be helpful to combine the findings of this paper and those of Herings et al. (2008) into the same context, in order to have a complete picture of the matter at hand. The key issues here obviously are the legal anti-piracy enforcement (captured by the generic cost factor of downloading) and the difference in taste in the society with regard to legal and illegal copies (captured by taste heterogeneity), and the way the society is influenced by these factors, both the consumers and the firm.

When the legal enforcement level is high (high cost factor of downloading), networks may fail to form and the firm in a lot of cases may have the possibility to deter the network using aggressive pricing or to ignore the network all together. In those cases, the firm has little worries. However, when the enforcement level is low, as we have seen in this paper, the firm is in much worse shape. Most of the network-deterring conditions will not be met and the firm has to accommodate the network and compete in price and sacrifice both on price and sales quantity, and thereby profit.

Only when the taste heterogeneity is low, the firm may have a chance to deter the network but only if it is ready to cover the entire market with aggressive pricing. This will only work if the level of legal enforcement is reasonably high. Needless to say, the firm should ensure the superior quality of the physical product, like in our newspaper market example.

At the other end of the scale, where the taste heterogeneity is so huge that the two plat-forms co-exist without competing for consumers leaving a section of market unserved is probably easier to conceive since most real-life markets are partially served.

Finally, it might be worth stressing that the total welfare in all of the possible market outcomes we have found in the model unambiguously benefits from low legal enforcement and low taste heterogeneity. The reason for the former is that low enforcement fosters a strong potential network, which either directly provides consumers' surplus by means of downloads or indirectly cuts deadweight loss by putting pressure on the price and thereby increasing sales quantity. The reason for the latter is simply the fact that taste heterogeneity can be considered as a sort of transportation cost that is subtracted from the consumers' surplus and going into no one else's surplus.

To sum up all the analysis that have been done around this model, it is the probably realistic to consider in most of the markets for information goods today the firms are accommodating networks. Furthermore, the analysis has given us some insights on how the market outcomes can be different when some of the factors we observe today were to change. It also gives suggestions to the impact on the firm's profits and the total welfare, as a result of these possible changes. 


\section{References}

[1] Bae S and J Choi (2006). A model of piracy. Information Economics and Policy 18: 303-320.

[2] Belleflamme P (2003). Pricing information goods in the presence of copying. In: R Nelson (Ed.), The Rate and Direction of Inventive Activity. Princeton University Press.

[3] Conner K and R Rumelt (1991). Software piracy: An analysis of protection strategies. Management Science 37: 125-139.

[4] Economides N and C Himmelberg (1995). Critical mass and network size with application to the US fax market. Discussion Paper no. EC-95-11. Stern School of Business, New York University.

[5] Farrell J and G Saloner (1985). Standardization, compatibility, and innovation. Rand Journal of Economics 16: 70-83.

[6] Farrell J and G Saloner (1986). Installed base and compatibility: innovation, product preannouncements, and predation. American Economic Review 76: 940-955.

[7] Fudenberg D and J Tirole (2000). Pricing a network good to deter entry. Journal of Industrial Economics 48: 373-390.

[8] Gayer A and O Shy (2003). Internet and peer-to-peer distributions in markets for digital products. Economics Letters 81: 197-203.

[9] Gayer A and O Shy (2006). Publishers, artists, and copyright enforcement. Information Economics 83 Policy 18: 374-384.

[10] Herings P, R Peeters and M Yang (2008) Competition against peer-to-peer networks. METEOR Research Memorandum RM/08/020, Maastricht University.

[11] Johnson W (1985). The economics of copying. Journal of Political Economy 93: 158174.

[12] Katz M and C Shapiro (1985). Network externalities, competition and compatibility. American Economic Review 75: 424-440.

[13] Katz M and C Shapiro (1986). Technology adoption in the presence of network externalities. Journal of Political Economy 94: 822-841.

[14] Liebowitz S (1985). Copying and indirect appropriability: photocopying of journals. Journal of Political Economy 93: 945-957. 
[15] Novos I and M Waldman (1984). The effects of increased copyright protection: an analytical approach. Journal of Political Economy 92: 236-246.

[16] Peitz M and P Waelbroeck (2006a). Piracy of digital products: A critical review of the theoretical literature. Information Economics and Policy 18: 449-476.

[17] Peitz M and P Waelbroeck (2006b). Why the music industry may gain from free downloading - The role of sampling. International Journal of Industrial Organization 24: $907-913$.

[18] Takeyama L (1994). The welfare implications of unauthorized reproduction of intellectual property in the presence of network externalities. Journal of Industrial Economics 42: 155-166.

[19] Zhang M (2002). Stardom, peer-to-peer and the socially optimal distribution of music. Mimeo. Sloan School of Management, MIT.

\section{A Solutions of the multi-platformed market structures}

\section{A.1 Fully-served market}

The consumer $z \in(0,1)$ that separates the consumers choosing for the firm from those choosing for the network, is herself indifferent between the two options. Moreover this consumer should weakly prefer the firm to the option not to acquire the product. So, for a second-stage equilibrium to generate the structure $[S / N]$, it should hold that, given the choices of everyone else,

$$
0 \leq U^{z}(p, S)=U^{z}(p, N) \quad \Longleftrightarrow \quad 0 \leq \beta-\tau z-p=\gamma-\tau(1-z)-\sigma(1-n(p))^{2},
$$

where $n(p)=1-z$, since the market is fully-served. Solving the inequality for $z$ gives us two solutions:

$$
z_{c . m .}(p)=\frac{\tau+\sqrt{\sigma(p+\gamma-\beta-\tau)+\tau^{2}}}{\sigma} \quad \text { and } \quad z_{m . n .}(p)=\frac{\tau-\sqrt{\sigma(p+\gamma-\beta-\tau)+\tau^{2}}}{\sigma}
$$

where we call the solution corresponding to the smaller network size $\left(z_{c . m}\right)$ the critical-mass network, and the solution corresponding to the larger network size $\left(z_{m . n .}\right)$ the maximum network. The solutions will only be real if $\sigma(p+\gamma-\beta-\tau)+\tau^{2} \geq 0$, which puts a condition on prices and will be discussed later in more detail. Moreover, $z \in(0,1)$ will be a necessary condition for the relevant parameters settings.

\section{A.2 Partially-served market}

The consumer $z^{S} \in(0,1)\left(z^{N} \in(0,1)\right)$ that separates the consumers choosing for the firm (network) from those choosing not to acquire any product, is herself indifferent between the 
two options. Moreover, this consumer prefers both the firm (network) and no acquisition to the option to acquire via the network (firm). We have a partially-served market if there is an interval of consumers that prefers no acquisition to acquisition, meaning that $z^{S}$ is less than $z^{N}$. So, for a second-stage equilibrium to generate the structure $[S / \emptyset / N]$, it should hold that, given the choices of everyone else,

$$
U^{z^{S}}(p, S)=U^{z^{S}}(p, \emptyset) \quad \text { and } \quad U^{z^{N}}(p, N)=U^{z^{N}}(p, \emptyset)
$$

and

$$
0 \leq z^{S}(p)<z^{N}(p) \leq 1
$$

The first two equations are equivalent to

$$
\beta-\tau z^{S}-p=0 \quad \text { and } \quad \gamma-\tau\left(1-z^{N}\right)-\sigma(1-n(p))^{2}=0 .
$$

where $n(p)=1-z^{N}$. Solving the first equation for $z^{S}$ and the latter for $z^{N}$ gives us one solution for $z^{S}$ :

$$
z^{S}(p)=\frac{\beta-p}{\tau},
$$

and two solutions for $z^{N}$ :

$$
z_{c . m .}^{N}=\frac{\tau+\sqrt{\tau^{2}+4 \sigma(\gamma-\tau)}}{2 \sigma} \quad \text { and } \quad z_{m . n .}^{N}=\frac{\tau-\sqrt{\tau^{2}+4 \sigma(\gamma-\tau)}}{2 \sigma} .
$$

Again, these solutions will only be real if $\tau^{2}+4 \sigma(\gamma-\tau) \geq 0$, which puts a condition on the relevant parameter settings and will be explained later in more detail.

\section{B Supports of Proposition 1-3}

\section{B.1 Proposition 1}

The following shows the feasible market structures for the high heterogeneity scenario $(\tau>$ $\gamma>\sigma)$ and the price range for each market structure.

$[S]$ requires that all consumers prefer the physical version to the digital version, including the consumer located at $x=1$ whose choice constitutes the binding condition,

$$
\beta-\tau-p \geq \gamma-\sigma \Longleftrightarrow p \leq \beta-\gamma+\sigma-\tau
$$

Hence, this is the maximum price with which the firm can grab hold of the whole market. A price any higher than this, the market will turn into $[S / N]$, which we will investigate little later on. Note that for this to be an equilibrium market structure, $\beta-\gamma+\sigma-\tau \geq 0$ is a necessary condition.

A necessary condition for the market structure $[N]$ to exist is that the consumer located at $x=0$ must have positive utility from downloading when everyone else is downloading. 
This requires $\gamma-\tau(1-0)-\sigma(1-1)^{2}>0 \Longleftrightarrow \gamma>\tau$, which is in conflict with the parameter setting in this scenario. We conclude, therefore, that in the high heterogeneity scenario $[N]$ does not exist.

$[\emptyset / N]$ though does exist in principle, and the condition for that is that the firm charges a price so high, i.e. when $p>\beta$, that no one buys the physical product from the firm. But this is not that interesting since in equilibrium $p>\beta$ will never be the case.

Next, under the market structure of $[S / N]$, there is a consumer located at $x=z$ who is indifferent from buying and downloading. The conditions that have to be fulfilled are the utilities of these choices are equal, positive, and the location of $z$ is within $(0,1)$. Formally,

$$
\begin{aligned}
\beta-\tau z-p & =\gamma-\tau(1-z)-\sigma z^{2} \\
0 & <z<1 \\
\beta-\tau z-p & \geq 0
\end{aligned}
$$

As explained in Appendix A, solving equation (1) yields two solutions for $z$ :

$$
z_{c . m .}(p)=\frac{\tau+\sqrt{\sigma(p+\gamma-\beta-\tau)+\tau^{2}}}{\sigma} \quad \text { and } \quad z_{m . n .}(p)=\frac{\tau-\sqrt{\sigma(p+\gamma-\beta-\tau)+\tau^{2}}}{\sigma},
$$

In order to guarantee the solution to be real, we need

$$
p \geq \beta-\gamma+\tau-\frac{\tau^{2}}{\sigma} .
$$

It can be easily seen that $z_{c . m .}>1$, as $\sqrt{\sigma(p+\gamma-\beta-\tau)+\tau^{2}} \geq 0$ and $\tau>\sigma$, and hence the critical mass equilibrium does not exist in this scenario.

Let us now look at the unique solution, the maximum network. Inequality (2) essentially has two components. The first component $0<z$ can be expressed as

$$
\begin{aligned}
& \frac{\tau-\sqrt{\sigma(p+\gamma-\beta-\tau)+\tau^{2}}}{\sigma}>0 \Longleftrightarrow \tau-\sqrt{\sigma(p+\gamma-\beta-\tau)+\tau^{2}}>0 \\
& \Longleftrightarrow \sigma(p+\gamma-\beta-\tau)<0 \Longleftrightarrow p<\beta-\gamma+\tau
\end{aligned}
$$

The second component $z<1$ can be expressed as

$$
\begin{aligned}
& \frac{\tau-\sqrt{\sigma(p+\gamma-\beta-\tau)+\tau^{2}}}{\sigma}<1 \Longleftrightarrow \sqrt{\sigma(p+\gamma-\beta-\tau)+\tau^{2}}>\tau-\sigma \\
& \Longleftrightarrow p+\gamma-\beta-\tau>-2 \tau+\sigma \Longleftrightarrow p>\beta-\gamma+\sigma-\tau
\end{aligned}
$$

Note that this coincides exactly the upper-bound price level of the market structure $[S]$. In other words, this is the price level where $[S]$ stops and $[S / N]$ starts. Finally, inequality (3) requires

$$
\beta-\tau \cdot \frac{\tau-\sqrt{\sigma(p+\gamma-\beta-\tau)+\tau^{2}}}{\sigma}-p \geq 0 \Longleftrightarrow \sqrt{\sigma(p+\gamma-\beta-\tau)+\tau^{2}} \geq \tau-\frac{\beta-p}{\tau} \cdot \sigma .
$$

To further simplify this inequality, we need to examine two cases. The first case is when $\tau-\frac{\beta-p}{\tau} \cdot \sigma<0$, which implies that inequality (3) would be trivially satisfied. It also means 
that $p<\beta-\frac{\tau^{2}}{\sigma}$. But for this to hold together with inequality (4), it must be that $\gamma>\tau$, which is in conflict with the parameter settings of this scenario. It leaves us the second case where $p \geq \beta-\frac{\tau^{2}}{\sigma}$, which implies,

$$
\sigma(p+\gamma-\beta-\tau)+\tau^{2} \geq \tau^{2}+\frac{\sigma^{2}}{\tau^{2}}(\beta-p)^{2}-2 \sigma(\beta-p) .
$$

Solving this inequality for $p$ yields,

$$
\beta-\frac{\tau}{2 \sigma}\left(\tau+\sqrt{\tau^{2}-4 \sigma(\tau-\gamma)}\right) \leq p \leq \beta-\frac{\tau}{2 \sigma}\left(\tau-\sqrt{\tau^{2}-4 \sigma(\tau-\gamma)}\right) .
$$

The condition on the left-hand side of inequality (5) is redundant because $\beta-\frac{\tau}{2 \sigma}(\tau+$ $\left.\sqrt{\tau^{2}-4 \sigma(\tau-\gamma)}\right) \leq \beta-\gamma+\sigma-\tau$. The condition on the right-hand side of inequality (5) is the upper bound of the price in $[S / N]$, as it is smaller than $\beta-\gamma+\tau .{ }^{9}$ Naturally for this price to be real, the condition $\tau^{2}-4 \sigma(\tau-\gamma) \geq 0$ must hold.

Finally, when the market structure $[S / \emptyset / N]$ prevails, there will be two indifferent consumers: the one who is indifferent between buying the physical product from the firm and not consuming at all, and the other who is indifferent from downloading from $\mathrm{P} 2 \mathrm{P}$ and not consuming at all. Let the former be located at $x=z^{S}$, then it must be that his utility of buying the physical product is equal to not consuming which is equal to 0 .

$$
\beta-\tau z^{S}-p=0 .
$$

Solving it for $z^{S}$ yields $z^{S}=\frac{\beta-p}{\tau}$. Let the consumer who is indifferent from downloading and not consuming be located at $x=z^{N}$, then we have

$$
\gamma-\tau\left(1-z^{N}\right)-\sigma\left(z^{N}\right)^{2}=0 .
$$

Solving it for $z^{N}$, we obtain two solutions: $z_{m . n .}^{N}=\frac{1}{2 \sigma}\left(\tau-\sqrt{\tau^{2}-4 \sigma(\tau-\gamma)}\right)$ and $z_{c . m .}^{N}=$ $\frac{1}{2 \sigma}\left(\tau+\sqrt{\tau^{2}-4 \sigma(\tau-\gamma)}\right)$, where, as before, $z_{m . n \text {. }}^{N}$ refers to the maximum network, and $z_{c . m}^{N}$. the critical mass network. Again it is easy to see that $z_{c . m}^{N}>1$, i.e. the critical mass network solution does not exist. ${ }^{10}$ The unique solution is then $z_{m . n .}^{N}$, which is only positive if $\tau>\gamma>\sigma$. This corresponds precisely to the parameter settings of this scenario. Furthermore, for the market structure $[S / \emptyset / N]$ to exist, the following condition has to be fulfilled.

$$
0<z^{S}<z_{m . n .}^{N}<1
$$

The left part of the inequality, $z^{S}>0$, is simply $\frac{\beta-p}{\tau}>0 \Longleftrightarrow p<\beta$. The right part of the inequality, $z_{m . n .}^{N}<1$, always holds. ${ }^{11}$ Now the middle part of inequality (6) is the only thing that puts a restriction on the price range of $[S / \emptyset / N]$. It requires $\frac{\tau-\sqrt{\tau^{2}+4 \sigma(\gamma-\tau)}}{2 \sigma}>\frac{\beta-p}{\tau} \Longleftrightarrow$ $\sqrt{\tau^{2}+4 \sigma(\gamma-\tau)}<\tau-\frac{(\beta-p) 2 \sigma}{\tau} \Longleftrightarrow p>\beta-\frac{\tau}{2 \sigma}\left(\tau-\sqrt{\tau^{2}-4 \sigma(\tau-\gamma)}\right)$.

\footnotetext{
${ }^{9}$ It is easy to verify that $\beta-\frac{\tau}{2 \sigma}\left(\tau-\sqrt{\tau^{2}}-4 \sigma(\tau-\gamma)\right)<\beta<\beta-\gamma+\tau$.

${ }^{10}$ It requires $\frac{\tau+\sqrt{\tau^{2}-4 \sigma(\tau-\gamma)}}{2 \sigma}>1 \Longleftrightarrow \sqrt{\tau^{2}-4 \sigma(\tau-\gamma)}>2 \sigma-\tau$. If $2 \sigma<\tau$, this is trivially true; and if $2 \sigma \geq \tau$, then it requires that $\tau^{2}-4 \sigma(\tau-\gamma)>\tau^{2}-4 \tau \sigma+4 \sigma^{2} \Longleftrightarrow \gamma>\sigma$, which is true.

${ }^{11}$ It requires $\frac{\tau-\sqrt{\tau^{2}-4 \sigma(\tau-\gamma)}}{2 \sigma}<1 \Longleftrightarrow \sqrt{\tau^{2}-4 \sigma(\tau-\gamma)}>\tau-2 \sigma$. If $\tau<2 \sigma$, this is trivially true; and if $\tau \geq 2 \sigma$, then it requires that $\tau^{2}-4 \sigma(\tau-\gamma)>\tau^{2}-4 \tau \sigma+4 \sigma^{2} \Longleftrightarrow \gamma>\sigma$, which is true.
} 


\section{B.2 Proposition 2}

The following shows the feasible market structures for the medium heterogeneity scenario $(\gamma>\tau>\sigma)$ and the price range for each market structure.

The market structure $[S]$ is still possible, and as conditions are the same as in the high heterogeneity scenario, so is the price range $p \leq \beta-\gamma+\sigma-\tau$. Any price higher than that, we will be in the market structure of $[S / N]$.

For the market of structure $[S / N]$, we will again have a unique solution, namely, the maximum network equilibrium $z_{m . n .}=\frac{\tau-\sqrt{\sigma(p+\gamma-\beta-\tau)+\tau^{2}}}{\sigma}$. This is because $z_{c . m} .>1$, hence the critical mass network equilibrium does not exist. Thus, for $[S / N]$ to exist, the following conditions have to be fulfilled.

$$
\begin{aligned}
& 0<z_{m . n .}<1 \\
& \beta-\tau \cdot z_{m . n .}-p \geq 0
\end{aligned}
$$

The left-hand side of inequality (7) requires

$$
\begin{aligned}
& \frac{\tau-\sqrt{\sigma(p+\gamma-\beta-\tau)+\tau^{2}}}{\sigma}>0 \Longleftrightarrow \sqrt{\sigma(p+\gamma-\beta-\tau)+\tau^{2}}<\tau \\
& \Longleftrightarrow \sigma(p+\gamma-\beta-\tau)<0 \Longleftrightarrow p<\beta-\gamma+\tau .
\end{aligned}
$$

The right-hand side of inequality (7) requires

$$
\frac{\tau-\sqrt{\sigma(p+\gamma-\beta-\tau)+\tau^{2}}}{\sigma}<1 \Longleftrightarrow \sqrt{\sigma(p+\gamma-\beta-\tau)+\tau^{2}}>\tau-\sigma \Longleftrightarrow p>\beta-\gamma-\tau+\sigma
$$

We will now show that inequality (8) does not further restrict the price. Inequality (8) is equivalent to $\sqrt{\sigma(p+\gamma-\beta-\tau)+\tau^{2}} \geq \tau-\frac{\sigma}{\tau}(\beta-p)$. In case the right-hand side of this inequality is negative, i.e. in case $p<\beta-\frac{\tau^{2}}{\sigma}$, the inequality is trivially satisfied. Otherwise, $p \geq \beta-\frac{\tau^{2}}{\sigma}$, then inequality (8) implies $\frac{\sigma}{\tau^{2}}(\beta-p)^{2}-(\beta-p)-(\gamma-\tau) \leq 0$, which is equivalent to $\beta-\frac{\tau}{2 \sigma}\left(\tau+\sqrt{\tau^{2}+4 \sigma(\gamma-\tau)}\right) \leq p \leq \beta-\frac{\tau}{2 \sigma}\left(\tau-\sqrt{\tau^{2}+4 \sigma(\gamma-\tau)}\right)$. Because $\gamma>\tau$, the lower bound on $p$ is less than $\beta-\frac{\tau^{2}}{\sigma}$ and therefore satisfied since we are considering the case $p \geq \beta-\frac{\tau^{2}}{\sigma}$. The upper bound is larger than $\beta$. Hence inequality (8) does not further restrict the price in either direction.

Now we show that the multi-platformed, partially-served market $[S / \emptyset / N]$ can not exist in the medium heterogeneity scenario. Consider the consumer who is indifferent from downloading and not consuming. Similar to the previous scenario, we solve for the condition of indifference and obtain two solutions: $z_{m . n .}^{N}=\frac{1}{2 \sigma}\left(\tau-\sqrt{\tau^{2}+4 \sigma(\gamma-\tau)}\right)$ and $z_{c . m .}^{N}=$ $\frac{1}{2 \sigma}\left(\tau+\sqrt{\tau^{2}+4 \sigma(\gamma-\tau)}\right)$. It is easy to see that, given $\gamma>\tau, z_{m . n .}^{N}<0$ and $z_{c . m .}^{N}>1$. This means that neither of these two solution exists.

Finally, opposite to the case in the high heterogeneity scenario, the market structure $[\emptyset / N]$ can not exist but $[N]$ can, because $\gamma>\tau$. The condition for this market structure to prevail is that the consumer located at $x=0$ prefers downloading from $\mathrm{P} 2 \mathrm{P}$ to buying from the firm. This implies $\gamma-\tau>\beta-p \Longleftrightarrow p>\beta-\gamma+\tau$. 


\section{B.3 Proposition 3}

The following shows the feasible market structures for the low heterogeneity scenario $(\gamma>$ $\sigma>\tau)$ and the price range for each market structure.

The market structure $[S]$ is once again similar as two previous scenarios, and the price range is $p \leq \beta-\gamma+\sigma-\tau$. At a higher price, the market structure will be $[S / N]$. Unlike the previous two scenarios, the market structure $[S / N]$ in this scenario is slightly more involved. In particular, both the critical mass network and the maximum network solutions exist, which causes multiple equilibria in the second stage.

We first look at the maximum network solution $z_{m . n .}$. The requirements for existence are as usual:

$$
\begin{aligned}
& 0 \leq z_{m . n .}<1 \\
& \beta-\tau \cdot z_{m . n .}-p \geq 0
\end{aligned}
$$

The left-hand side of inequality (9) requires, as in the medium heterogeneity scenario, $p \leq$ $\beta-\gamma+\tau$. This is the upper-bound price for the market structure $[S / N]_{m . n .}$. The right-hand side, however, leads to a different condition due to the fact that $\tau<\sigma$.

$$
\frac{\tau-\sqrt{\sigma(p+\gamma-\beta-\tau)+\tau^{2}}}{\sigma}<1 \Longleftrightarrow \sqrt{\sigma(p+\gamma-\beta-\tau)+\tau^{2}}>\tau-\sigma .
$$

Since $\sigma>\tau$ in this scenario, the condition is satisfied whenever the square root exists, which is when $p>\beta-\gamma+\tau-\frac{\tau^{2}}{\sigma}$. Similar to the medium heterogeneity scenario, inequality (10) does not further restrict the prices.

The lower-bound price of $[S / N]_{m . n}$. is therefore $\beta-\gamma+\tau-\frac{\tau^{2}}{\sigma}$, which is below the upperbound price of $[S], \beta-\gamma+\sigma-\tau$. This can be shown by supposing if the opposite were true, namely $\beta-\gamma+\tau-\frac{\tau^{2}}{\sigma}>\beta-\gamma+\sigma-\tau$. After rearranging, we get $2 \tau-\frac{\tau^{2}}{\sigma}-\sigma>0$. Multiplying both sides by $\sigma$ and rearranging yields $(\tau-\sigma)^{2}<0$, which is clearly impossible. Therefore the price in the market structure $[S / N]_{m . n}$. satisfies $\beta-\gamma+\tau-\frac{\tau^{2}}{\sigma}<p<\beta-\gamma+\tau$.

Furthermore, we need to investigate the critical mass solution $z_{c . m}$. in this scenario. The requirements for existence are similar to those of $z_{m . n .}$, namely,

$$
\begin{aligned}
& 0 \leq z_{c . m .}<1 \\
& \beta-\tau \cdot z_{c . m .}-p \geq 0
\end{aligned}
$$

The left-hand side of inequality (11) is trivially satisfied. The right-hand side can be simplified to $p<\beta-\gamma+\sigma-\tau$. Inequality (12) is equivalent to

$$
\sqrt{\sigma(p+\gamma-\beta-\tau)+\tau^{2}} \leq \frac{\sigma}{\tau}(\beta-p)-\tau .
$$

This condition holds only if the right-hand side of this inequality is non-negative, that is $p \leq \beta-\frac{\tau^{2}}{\sigma}$, and

$$
\frac{\sigma}{\tau^{2}}(\beta-p)^{2}-(\beta-p)-(\gamma-\tau) \geq 0,
$$


where the latter inequality is equivalent to

$$
p \leq \beta-\frac{\tau}{2 \sigma}\left(\tau+\sqrt{\tau^{2}+4 \sigma(\gamma-\tau)}\right) \quad \text { or } \quad p \geq \beta-\frac{\tau}{2 \sigma}\left(\tau-\sqrt{\tau^{2}+\sigma(\gamma-\tau)}\right) .
$$

Since $\gamma>\tau$, the right-hand side of the second inequality exceeds $\beta$ and hence cannot be satisfied - leaving the first inequality to be satisfied. Moreover, the right-hand side of the first inequality is less than $\beta-\frac{\tau^{2}}{\sigma}$, hence inequality (12) is satisfied if and only if

$$
p \leq \beta-\frac{\tau}{2 \sigma}\left(\tau+\sqrt{\tau^{2}+4 \sigma(\gamma-\tau)}\right) .
$$

Now we need to see if this poses a further restriction on the upper-bound price. Since $\gamma>\tau$, we know that $\sqrt{\tau^{2}+4 \sigma(\gamma-\tau)}>\tau$. Because $\tau<\sigma$, it is then easy to see that $\beta-\frac{\tau}{2 \sigma}\left(\tau+\sqrt{\tau^{2}+4 \sigma(\gamma-\tau)}\right)>\beta-\tau$. But because $\gamma>\sigma$, we also know that $\beta-\gamma+$ $\sigma-\tau<\beta-\tau$. This implies that inequality (12) does not pose further restrictions on the upper-bound price. Therefore, the price boundaries for the market structure $[S / N]_{c . m}$. are $\beta-\gamma+\tau-\frac{\tau^{2}}{\sigma}<p<\beta-\gamma-\tau+\sigma$.

Finally, the market structure $[N]$ is the same as in the medium heterogeneity scenario, namely, $p>\beta-\gamma+\tau$. 